\title{
Scattering From Controlled Defects in Woodpile Photonic Crystals
}

\author{
Stefan Aeby, Geoffroy J. Aubry,* Nicolas Muller, and Frank Scheffold**
}

Photonic crystals display partial or full band gaps that become more pronounced with rising refractive index contrast. However, imperfections in the material cause light scattering and extinction of the interfering propagating waves. Positive as well as negative defect volumes may contribute to this kind of optical perturbation. In this study, 3D woodpile photonic crystals are fabricated and characterized with a pseudo-bandgap for near-infrared optical wavelengths. By direct laser writing, defects are introduced in the periodic structure at selected positions. It is shown that defect scattering can be modeled by considering the difference between the disordered and the regular structure. The findings pave the way toward better control and understanding of the role of defects in photonic materials that will be crucial for their usability in potential applications.

imperfections in manufacturing and the resulting defect scattering..$^{[1,8]}$

Photonic crystals (PCs) are metamaterials fabricated from mesoscopic building blocks by self-assembly or by lithography. ${ }^{[9-14]}$ In contrast to the case of atomic or molecular crystals, these building blocks are not identical. Therefore, all photonic crystal materials possess some intrinsic degree of disorder due to surface roughness, size dispersion, stress-induced deformations, in addition to defects, stacking faults, or crystal grain boundaries. The influence of disorder is often so strong that the interaction of the propagating wave with the periodic Bragg planes competes with scattering on

\section{Introduction}

Dielectric materials with a periodic variation of the refractive index display photonic stop bands for a monochromatic optical wave propagating in specific directions. ${ }^{[1]}$ For a perfect crystal, destructive interference always leads to vanishing transmission in the stop band irrespective of the refractive index contrast. ${ }^{[2]}$ The Bragg length $L_{\mathrm{B}}$ is a measure for the number of crystal layers penetrated by the incident beam and it is directly related to the scattering strength of the crystal layers. ${ }^{[3,4]}$ Above a certain threshold refractive index contrast, ${ }^{[1,5]}$ the propagation of light can be inhibited in all directions, opening a full photonic bandgap (PBG) with a Bragg length $L_{\mathrm{B}}$ eventually becoming comparable to the lattice constant. Bandgaps in one and 2D photonic crystals are now widely employed in applications such as supercontinuum fiber lasers ${ }^{[6]}$ and for data processing using optical modules based on 2D silicon photonic crystal technology. $\left.{ }_{[}\right]$Technological applications for 3D PBG bandgap materials offer great potential in lasing, wave-guiding, light-harvesting and even optical computers. In practice these 3D-functionalities remained elusive, mainly due to the role of

S. Aeby, Dr. G. J. Aubry, Dr. N. Muller, Prof. F. Scheffold

Department of Physics

University of Fribourg

Fribourg 1700, Switzerland

E-mail: geoffroy.aubry@unifr.ch; frank.scheffold@unifr.ch

Dr. N. Muller

iPrint Institute, HEIA-FR

HES-SO University of Applied Sciences and Arts Western Switzerland

Fribourg 1700, Switzerland

The ORCID identification number(s) for the author(s) of this article can be found under https://doi.org/10.1002/adom.202001699.

DOI: 10.1002/adom.202001699 similar length scales. In early work on opal photonic crystals, the manifestation of intrinsic defects has been discussed and a plethora of studies found that it is nearly impossible to fabricate perfect crystals made by self-assembly of colloids. ${ }^{[15]}$ As a consequence, artificial opals of polystyrene spheres or air holes in $\mathrm{TiO}_{2}$ (titanium dioxide) display diffuse multiple-scattering in tandem with (multiple) Bragg reflection. ${ }^{[16,17]}$ Similar observations have been made for quasi-crystals. ${ }^{[18]}$ Improved selfassembly protocols and lithography have led to higher quality photonic crystals, but despite the progress made, imperfections still play a significant role. ${ }^{[13,19]}$ While disorder in photonic crystals is often considered a nuisance, it also highlights the rich and fascinating interplay between defect states, wave tunneling and percolation, random diffuse scattering, and directed Bragg scattering of light. ${ }^{[20-25]}$ Moreover, the interaction between the band structures and defect scattering might facilitate the observation of other critical coherent transport phenomena such as Anderson localization of light. In a seminal paper published in 1987, Sajeev John suggested the presence of localized defect states close to the band edge of a photonic crystal due to multiple scattering and a reduced density of states. ${ }^{[26]}$ Finally, defect states can be introduced in a photonic crystal deliberately to implement a particular function, such as for optical sensing applications, lasing, or optical circuitry. ${ }^{[1,27]}$ Controlling and understanding the role of defects and disorder in photonic crystals is thus of paramount importance.

Here, we report on a study about intrinsic and added defects in $\mathrm{PCs}^{[28]}$ fabricated in a polymer resist by direct laser writing (DLW), see Figure 1. The tight control over the position and size of the defects we have sets our study apart from earlier work on intrinsic ${ }^{[29]}$ or added defects in opals. ${ }^{[30,31]}$ We moreover model the defects induced scattering of these structures without any fit parameter. 

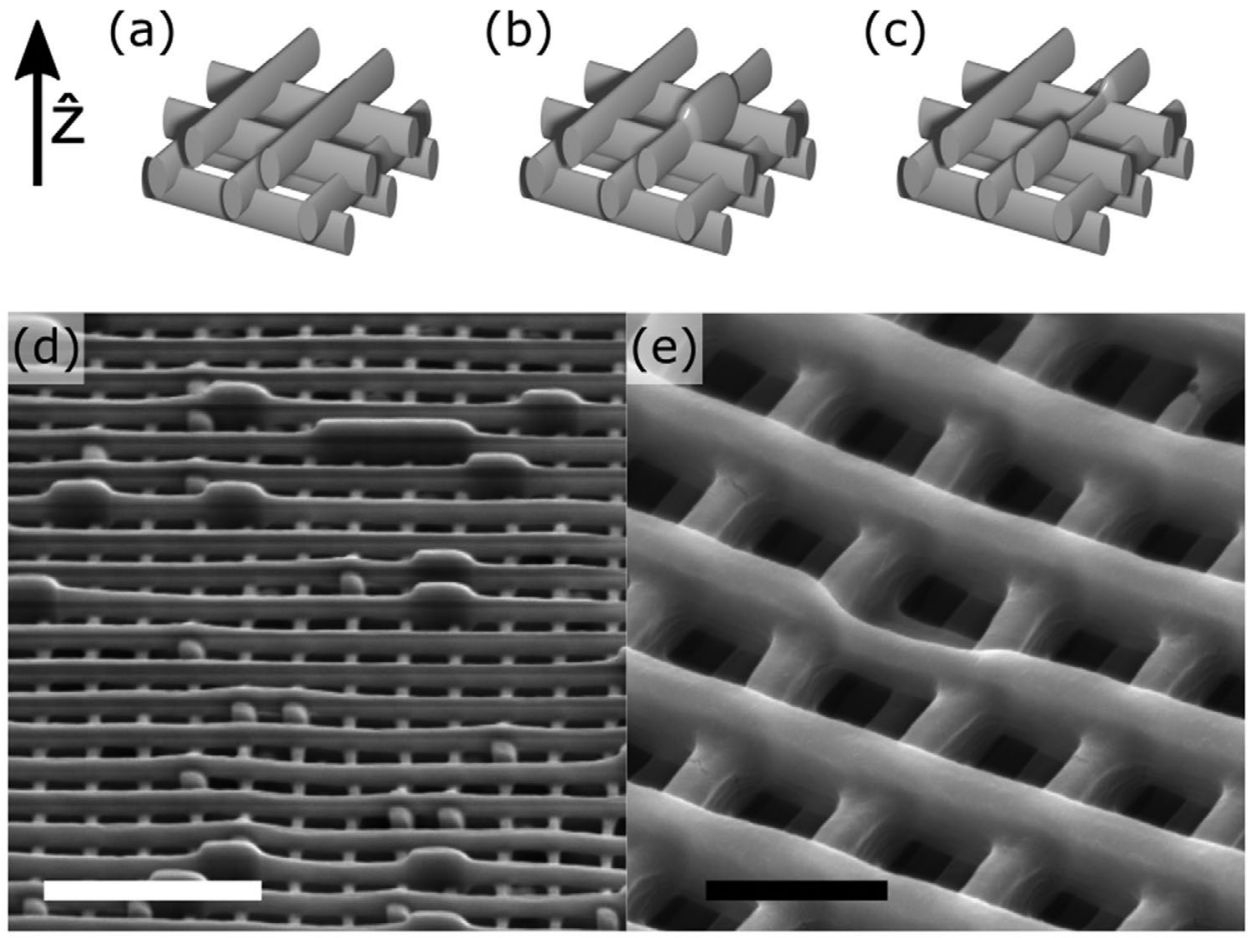

Figure 1. Computer generated renderings of a) a woodpile structure, b) a woodpile structure with positive defects, c) a woodpile structure with negative defects. Scanning electron micrograph of polymer woodpile structures fabricated by DLW, with d) positive (rod cross section area $+280 \%$, image taken at an angle of $\left.52^{\circ}\right)$, and (e) negative defects $\left(-64 \%, 45^{\circ}\right.$ tilted). Scale bars are $5 \mu \mathrm{m}$ for (d), $1 \mu \mathrm{m}$ for (e).

\section{Results}

By DLW in a polymer resist ${ }^{[12,32-35]}$ (Photonic Professional, Nanoscribe, Germany), we fabricate high-quality woodpile (WP) photonic crystals displayed in Figure 1d,e. For optimal results, we use the IP-Dip photoresist (Nanoscribe, Germany), refractive index $n_{\text {IP-Dip }}=1.53$. $^{[36]}$ We add defects to our crystals to probe the effect of disorder on the photonic properties of the PCs. We introduce two types of defects: positive and negative defects, see Figure 1, and we place these defects uniformly over the sample volume with a variable defect number density $\rho$. We position the defects randomly, constrained by the condition that two defects cannot overlap.

The basic WP-structures are composed of horizontal arrays of parallel rods, where $d$ denotes the in-plane distance between two rods. Alternating layers are rotated by $90^{\circ}$ and shifted by $d / 2$ every two layers. Hence, the structure repeats itself over a distance $d_{z}$ corresponding to four layers in the stacking direction $\hat{z}$. We use a ratio $d_{z} / d=\sqrt{2}$ which results in a face-centered-cubic-lattice (FCC, see Figure S3, Supporting Information). The rods of adjacent layers slightly overlap, as indicated in Figure 1a-c. For our samples, the in-plane-distance between rods, which is equal to the rod-segment length, is set to $d=1.2 \mu \mathrm{m}$. We note that smaller in-plane-distances can be readily achieved, ${ }^{[12]}$ but it would not add benefit to the current work. All the samples have a total size of $70 \times 70 \times 8.5 \mu \mathrm{m}^{3}$ which corresponds to five lattice constants of the conventional cubic unit cell of the FCC lattice (20 layers of rods) in the $\hat{z}$ direction (i.e., perpendicular to the rods long axis, see Figure 1a). Note that one rod-segment corresponds to the primitive unit cell lattice constant, see Figure S3, Supporting Information. By locally enlarging (reducing) the thickness of a rod-segment, Figure $1 \mathrm{~b}, \mathrm{c}$, we create positive (negative) defects. We denote the relative increase or decrease of the rod cross sectional area, or volume, with $\kappa$. We control the thickness of the rods by setting the power of the DLW-laser below or above the default power used to fabricate the woodpile crystal.

Due to the asymmetric shape of the DLW focal volume, set by the point-spread function of the DLW-microscope objective, the cross-section of the rods is elliptical with an aspect ratio of nearly three. For each laser power used, we measure the rod cross sectional area with a scanning electron microscope (SEM); see Experimental Section. For the crystal, we find for the long axis $a=0.42 \mu \mathrm{m}$, and the short axis $b=0.15 \mu \mathrm{m}$.

Figure 2 shows the reflectance and transmittance spectra recorded for a series of samples. The measurements were performed by Fourier transform infrared spectroscopy (FTIR, Bruker Vertex 70, and Hyperion Spectrometer). As we use a Cassegrain mirror-objective in the FTIR-microscope, the light is transmitted and detected along a hollow cone with an acceptance angle between $\theta_{\min }=15^{\circ}$ and $\theta_{\max }=30^{\circ}$ with $\theta_{\text {mean }}=22.5^{\circ}$ compared to the $\Gamma-\mathrm{X}_{z}$-direction of the crystal (see Figure S5, Supporting Information for the FCCBrillouin zone scheme). We measure the spectra averaged over an area of about $40 \times 40 \mu \mathrm{m}^{2}$ and consider wavelengths between 1.5 and $2.7 \mu \mathrm{m}$ while for wavelengths larger than $2.7 \mu \mathrm{m}$, the polymer absorbs light (for details see Experimental Section). We use a silver-coated mirror as a reference for the reflectance spectra. To calibrate the transmittance, we 


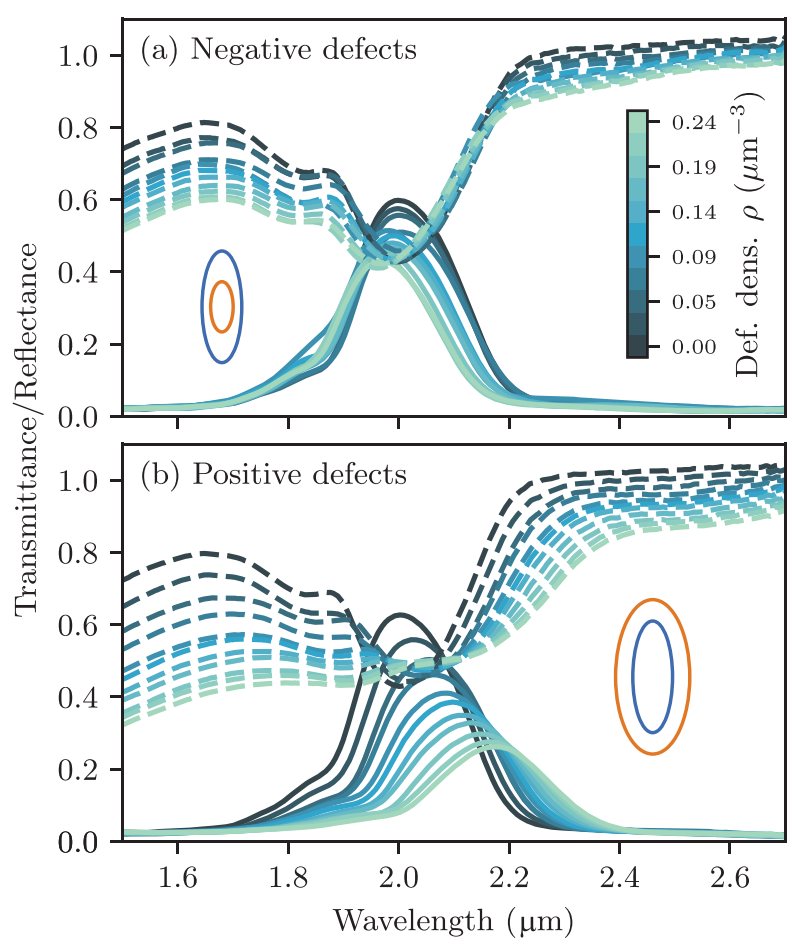

Figure 2. Experimental reflectance (solid lines) and transmittance (dashed lines) of woodpile structures, thickness $L=8.5 \mu \mathrm{m}$, with different defect number densities. The density ranges from 0 to $0.24 \mu^{-3}$. a) Spectra for negative defects ( $\kappa=-74 \%$ cross section surface), b) for positive $(\kappa=+158 \%)$. The blue ellipses in inset show the standard dimensions of the WP-rods, while the orange ellipses show the dimensions the negative (a) and positive (b) defects. Each curve is averaged over three to five samples to take into account the sample to sample variations.

measure the spectrum of the bare glass substrate next to the samples. For each set of fabrication parameters, we produce several samples, and each curve in Figure 2 represents an average over three to five different sample realizations. The darkest lines show the spectra of the crystalline samples, that is, without any added defects. The transmittance (reflectance) displays a profound dip (peak) indicating the presence of a pseudo-gap in the chosen incident direction. We find that by increasing the number density $\rho$ of defects, the peak of the reflectance and the dip of the transmittance become less marked meaning that the quality of the bandgap is affected by the presence of defects. In our experiments, we see that the pseudo-band gap disappears continuously with no clear threshold. The situation would be different for a full PBG where defect states are initially isolated, and then percolate once the defect density exceeds a certain threshold. ${ }^{[21]}$ Interestingly, we also observe that the peaks are shifted to higher (lower) wavelengths when increasing the density of positive (negative) defects. To study this effect quantitatively, we plot in Figure 3 the position of the reflectance maxima for different defect sizes and defect densities against the polymer filling fraction $\phi$ of each sample (see Experimental Section for the method used to estimate the filling fraction). Interestingly, we find that all the data collapse on a master curve. The pseudo-gap center wavelength increases roughly linearly with

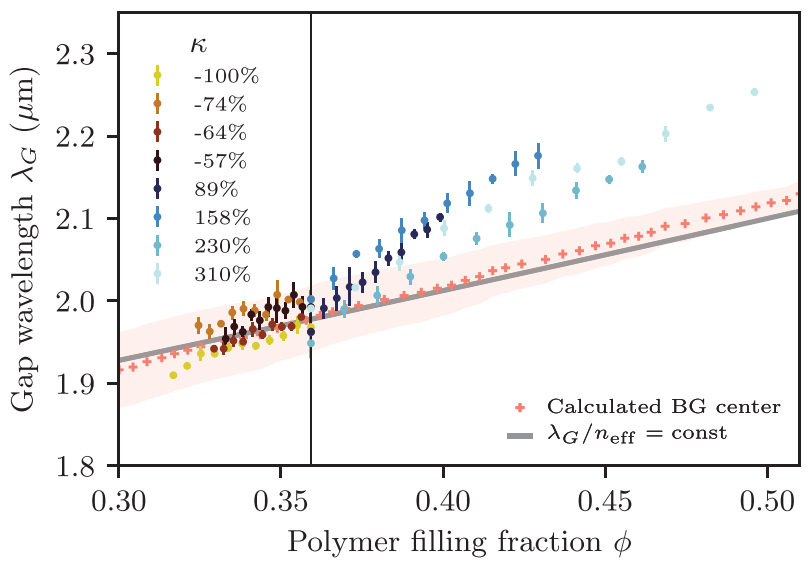

Figure 3. Center position (circles) of the reflection peak marking the position of the photonic pseudo-gap in the presence of defects. Each color corresponds to a different defect size. The values of $\kappa$ denote the volume of the defects in units of the unperturbed rod-segment. $-100 \%$ indicates a missing link, $+310 \%$ means the interstitial space between parallel rods is entirely filled when two defects are adjacent, marking the two extreme cases. The error bars correspond to the standard deviation of the different realizations for equal fabrication parameters. The vertical line indicates the filling fraction of the WP crystal. In red: pseudo-gap span (area) and center (crosses) calculated with MPB for the WP crystal with elliptical rods as a function of the rods filling fraction keeping the rods aspect ratio constant. The grey line shows the expected scaling of the gap wavelength assuming a linear dependency with the Maxwell-Garnet effective refractive index $n_{\text {eff. }}$

$\phi$, independent of whether the defect volume or number density is varied to achieve a certain $\phi$-value.

We corroborate this finding's accuracy by band structure calculations with MPB (MIT Photonic Bands) ${ }^{[37]}$ for WP photonic crystals with different filling fractions. For all the filling fractions, the calculations, shown as red crosses in Figure 3, were performed for elliptical rods with a constant aspect ratio of $a / b=0.42 / 0.15=2.8$. To accurately compare the simulations with the measurements using a Cassegrain-objective, we proceed as follows. We calculate the intersections of the gaps for all the directions making an angle $\theta_{\text {eff }}$ relative to the $\Gamma-\mathrm{X}_{z}$ direction, where $\theta_{\text {eff }}$ is defined by the incident direction (due to the Cassegrain objective, $\theta_{0}=22.5^{\circ}$ ) and the refraction at the interface (due to the effective medium, calculated for each filling fraction using the Maxwell-Garnett formula), for details see Experimental Section.

Our results suggest that with increasing filling fraction $\phi$, the MPB-value of $\lambda_{G}$ increases and its evolution follows the Maxwell-Garnett effective refractive index mixing formula. ${ }^{[38]}$ The latter predicts a roughly linear increase of the effective refractive index $n_{\text {eff }}$ with $\phi$ over the studied range, see the gray line in Figure 3 . This shift of $\lambda_{G}$ can be explained as follows. The reflectance maximum is due to Bragg backscattering at a scattering angle $\Theta \simeq 180^{\circ}$ where the momentum transfer $\vec{q}=2 \vec{k} \sin [\Theta / 2] \equiv \vec{G}$ matches a reciprocal lattice vector $\vec{G}$. Our results suggest that the wave number, and thus the phase delay, is defined by the effective medium $|k|=2 \pi n_{\mathrm{eff}} / \lambda_{\mathrm{G}}$. Thus, the maxima $\lambda_{G}$ scale with the effective refractive index of the medium $n_{\text {eff }}$ such that $\lambda_{G} / n_{\text {eff }}$ remains constant. Interestingly, we find this effect to be surprisingly robust against defect scattering. 


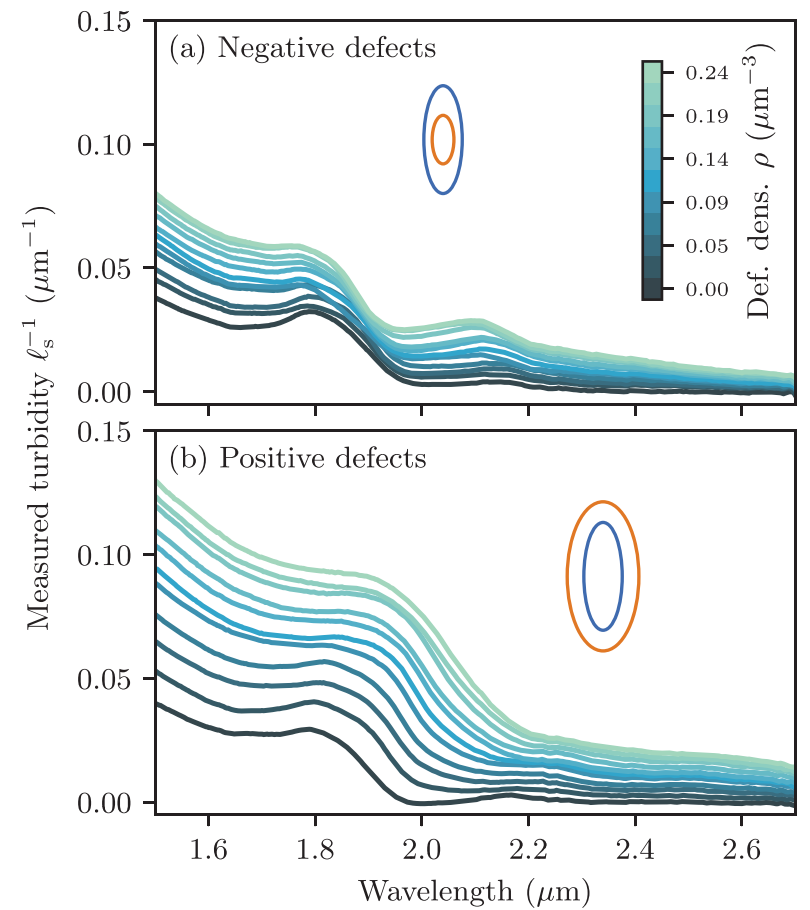

Figure 4. Turbidity (inverse of the scattering mean free path) of woodpile structures with a) negative and b) positive defects. The different colors of the curves encode the defect number density $\rho$ in $\mu \mathrm{m}^{-3}$ in the sample. The samples are the same as in Figure 2.

Next, we study the scattering by the induced defects. We use a modified Beer-Lambert's law to extract the scattering mean free path $\ell_{\mathrm{s}}$ from the simultaneous reflection $R$ and transmission $T$ measurements of the PC,

$T(L)+R(L)=\alpha \exp \left(-L / \ell_{\mathrm{s}}\right)$

where $L$ is the thickness of the sample. This relation is valid before the onset of diffraction and for negligible absorption. ${ }^{[39,40]}$ Both conditions are met in our case over the range of wavelengths studied; see also Experimental Section for more details. The additional factor $\alpha$ in Equation (1) takes account of systematic errors in the calibration procedure. The bare substrate and the sample covered substrate deviate in two ways. First, the sample acts as an anti-reflection coating since the effective index $n_{\text {eff }} \simeq 1.17$ lies in between glass and air, which leads to an increased optical transmission $T$. Second, the sample thickness, $L \simeq 8.5 \mu \mathrm{m}$, is only a small multiple of the wavelength, which results in Fabry-Pérot interferences between the paths reflected by the sample top and the WP/substrate interface. As a consequence, we observe slow oscillations in $T(\lambda)$ which complicates proper calibration. To achieve a model independent calibration, for each set of samples, we take averages of $T+R$ between $\lambda=2.45$ and $2.55 \mu \mathrm{m}$. Over this wavelength range, the $T(\lambda)+R(\lambda)$ curves are flat for the crystal structure and thus $T+R \simeq \alpha$, see also figure S7, Supporting Information. We find typical values of $\alpha$ between 1.037 and 1.055. From the data shown in Figure 2, and using Equation (1), we extract $\ell_{\mathrm{s}}$ as a function of $\lambda$ as shown in Figure 4.
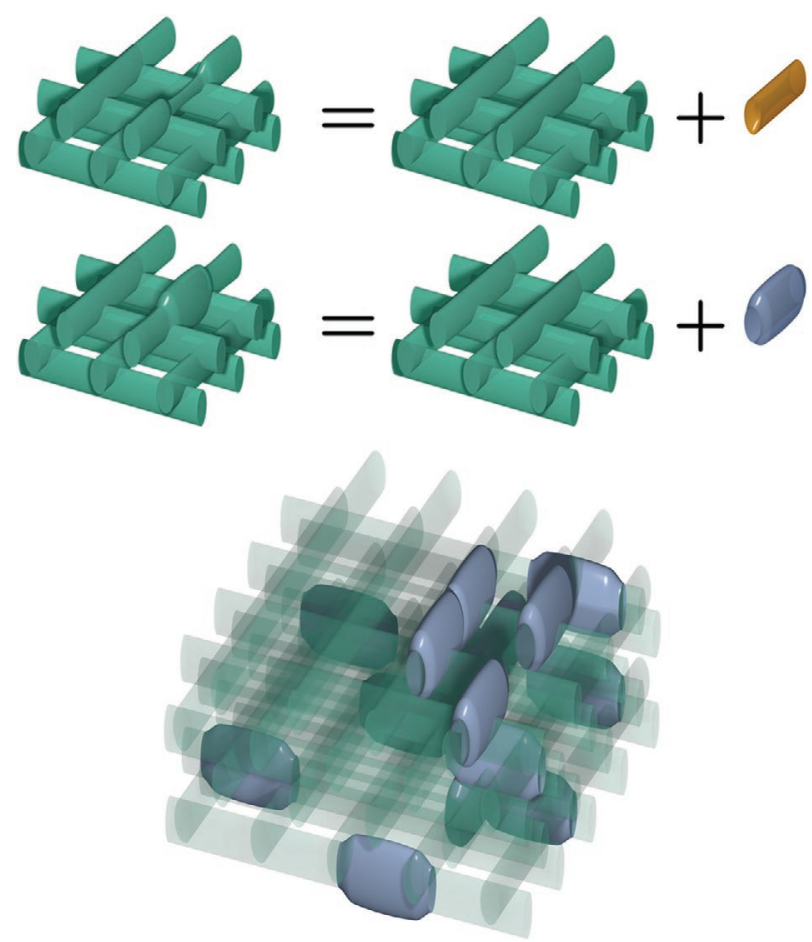

Figure 5. The measured turbidity of the samples is the sum of two contributions, the intrinsic turbidity of the woodpile crystals (due to DLW imperfections) on one hand, and the induced turbidity due to the artificially added defects on the other hand.

\section{Discussion}

We first note that the turbidity of the WP crystals, given by the inverse of the scattering mean free path, $1 / \ell_{\mathrm{s}}$, is finite, and it rises in the low-wavelength regime. The residual scattering from WP crystals, observed previously in ref. [12], is caused by intrinsic roughness, small displacements, undulations, and deformations in the crystal. The turbidity of a crystal in the presence of artificially added defects is therefore determined by defect scattering and by intrinsic scattering. In Figure 5, we illustrate how we can understand the scattering from our materials as a sum of scattering from the WPs and the added defects. If we assume that these two effects contribute independently, we can express the measured turbidity $1 / \ell_{\mathrm{s}}$ as follows ${ }^{[41]}$

$\frac{1}{\ell_{\mathrm{s}}}=\frac{1}{\ell_{\mathrm{s}, 0}}+\frac{1}{\ell_{\mathrm{s}, \text { ind }}}$

where $1 / \ell_{\mathrm{s}, 0}$ corresponds to the intrinsic scattering and $1 / \ell_{\mathrm{s}, \text { ind }}$ to the artificial defects scattering. The same expression can be derived in the frame of the classical theory for the resistivity of ordinary metals. ${ }^{[42]}$ In the latter case, the resistivity is controlled by the scattering mean free path of electrons: $1 / \ell_{\mathrm{s}, 0}$ corresponds to the residual resistivity contribution, due to intrinsic defects, and $1 / \ell_{\mathrm{s} \text {,ind }}$ to the temperature dependent contribution $\propto T$, also known as the Wiedemann-Frantz law, due to electron-phonon scattering.

Both for negative (Figure 4a) and positive (Figure 4b) defects, we see that the induced turbidity increases with the defect 


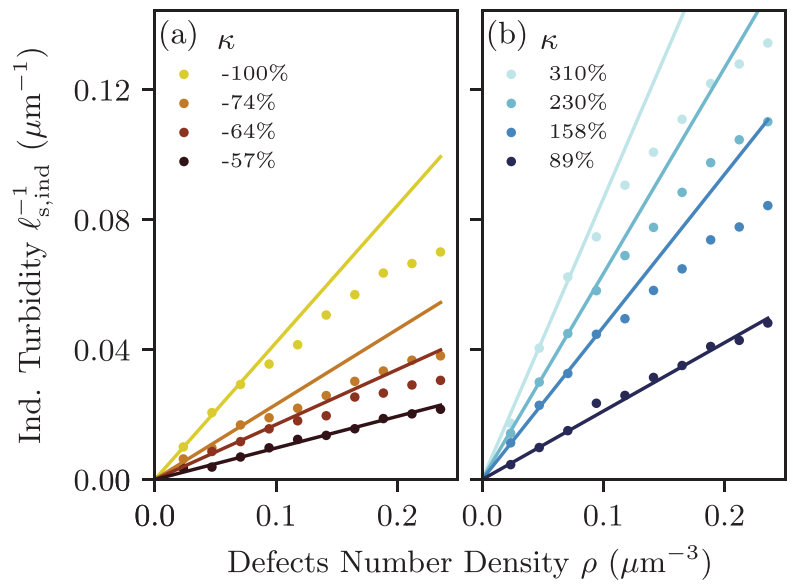

Figure 6. Induced turbidity for different defect volumes measured at $\lambda_{0}=1.57 \mu \mathrm{m}$ for a) negative and b) positive defects as a function if the defects number density. Each color corresponds to a different relative change of the rod cross section for a single defect with respect to the default rod. Lines are linear fits done on the first points.

number density $\rho$. In Figure 6, we plot $1 / \ell_{\mathrm{s}, \text { ind }}\left(=1 / \ell_{\mathrm{s}}-1 / \ell_{\mathrm{s}, 0}\right)$ at a selected wavelength $\left(\lambda_{0}=1.57 \mu \mathrm{m}\right)$ as a function of the defect number density. We plot the data for negative (Figure 6a) and positive (Figure 6b) defects of different size. The induced turbidity increases linearly with $\rho$ for small defect number densities, as expected for independent random scatterers. For higher concentrations, positional correlations and proximity effects lead to deviations from the linear dependence. ${ }^{[43-45]}$ This sound result supports that using Equation (2) and Equation (1) to determine $1 / \ell_{\mathrm{s} \text {, ind }}$ is justified. The turbidity of a medium consisting of identical uncorrelated scatterers is given by $1 / \ell_{\mathrm{s}}=\rho \sigma$, where $\sigma$ denotes the total scattering cross-section of a scatterer. Therefore, the initial slope of $1 / \ell_{\mathrm{s}}(\rho)$ is a measure for the total scattering cross section of defects

$\frac{1}{\ell_{\mathrm{s}, \text { ind }}}=\rho \sigma_{\text {defects }}$

In Figure 7 , we report the values for $\sigma_{\text {defects }}$ extracted in the low- $\rho$ regime as a function of $r_{\mathrm{d}}=\sqrt{a_{\mathrm{d}} b_{\mathrm{d}}}$ where $a_{\mathrm{d}}$ and $b_{\mathrm{d}}$ are the long and short axis of the defect rod-segments. For all the samples studied, we report data for five different wavelengths.

Inspired by earlier work on disordered opals, ${ }^{[29]}$ we model the defect scattering in the frame of the first Born, also known as Rayleigh-Gans-Debye (RGD) scattering, approximation. We hypothesize that we can treat the WP crystal as an effective, homogeneous background medium, and scattering arises from the local density differences, as shown in Figure 5. To this end, we calculate the known RGD-expression for the total scattering cross-section of a hollow cylinder $C_{\text {sca,hc }}$ with a length $d=1.2$ $\mu \mathrm{m}$ equal to a rod segment. ${ }^{[46]}$

For simplicity, in our calculations, we consider scattering from hollow cylinders with $n_{\text {IP-Dip }}$ in air $\left(n_{\text {air }}=1\right)$, and limiting radii $r_{\mathrm{d}}=\sqrt{a_{\mathrm{d}} \cdot b_{\mathrm{d}}}, \quad r=\sqrt{a \cdot b}=0.25 \mu \mathrm{m}$. For positive defects $r_{\mathrm{d}}>r$, while for negative defects $r_{\mathrm{d}}<r$. We explicitly take into

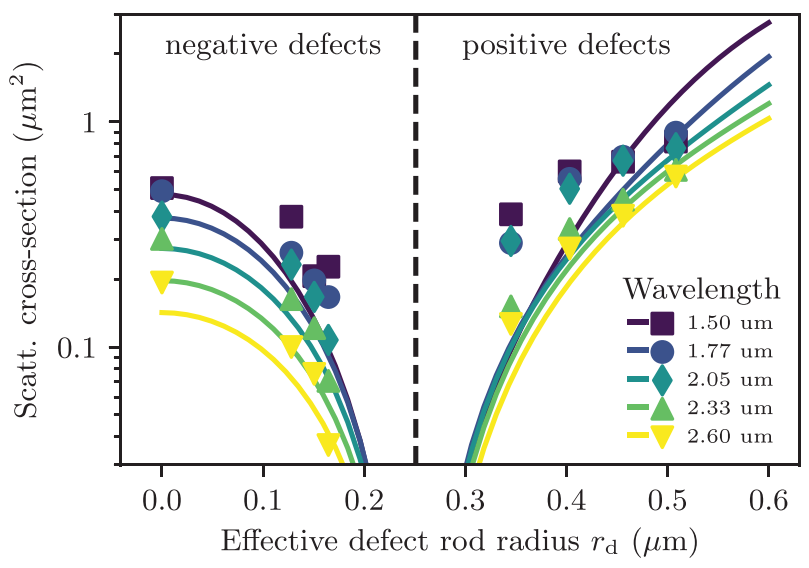

Figure 7. Total scattering cross-section of defect scatterers. Symbols show $\sigma_{\text {defects }}$ extracted from the slopes of the curves $\left(\rho \ell_{\mathrm{s}, \text { ind }}\right)^{-1}$ (solid lines shown in Figure 6). The effective radius of the defect rod-segments is denoted by $r_{\mathrm{d}}=\sqrt{a_{\mathrm{d}} \cdot b_{\mathrm{d}}}$. The lines show the scattering cross sections of the hollow cylinders $C_{\text {sca, hc }}$ with limiting radii $r_{d}$ and $r=\sqrt{a \cdot b}=0.25 \mu \mathrm{m}$ (the standard rod size in the crystal, vertical dashed line).

account the optical geometry imposed by the Cassegrain objective. Therefore, we calculate $C_{\text {sca,hc }}$ for an angle between the incoming beam and the cylinder axis $\zeta=90^{\circ}-<\theta_{\text {eff }}>=71.3^{\circ}$. $\theta_{\text {eff }}$ is the angle after refraction by the effective medium $\left(n_{\text {eff }}\right)$ when the incident angle is $22.5^{\circ}$. We calculate $<\theta_{\text {eff }}>$ by averaging $\theta_{\text {eff }}$ over the different $n_{\text {eff }}$ obtained by varying the filling fraction over the experimental range $(0.3<\phi<0.5)$. The effective refractive index $n_{\text {eff }}$ also enters via the effective wavenumber $k_{\mathrm{eff}}=2 \pi n_{\mathrm{eff}} / \lambda_{0}$.

In contrast to earlier studies on disordered opals, ${ }^{[16,29]}$ our entire modeling predictions (shown as lines in Figure 7) are fit-parameter free. Overall, we find a good agreement between the data and the model: the calculated scattering cross-sections follow the trend of the experimental data. The agreement between theory and experiment is nearly quantitative for the higher wavelengths where the intrinsic scattering is negligible, and the transmission of the unperturbed crystal is high, signaling a trend toward an effective homogeneous medium.

In conclusion, in this study, we have quantified the effect of intrinsic and induced defects on photonic crystals' optical properties. We demonstrate that both the bandgap position and its quality are simultaneously affected by defect scattering. The study presented in this work gives essential guidelines on how to quantify and model defect scattering. Our study also provides a modeling framework for diffuse scattering in PCs that lays the ground toward more complex disordered PCs based photonic materials. Such materials, based on higher refractive index building blocks, could become crucial to reach conditions for Anderson localization of light. ${ }^{[47]}$ To achieve this goal, one will need to increase the refractive index contrast of these structures, for example by using a double inversion technique. ${ }^{[48]}$

\section{Experimental Section}

Rod Size Measurement: The lateral size of the rods, or the short axis of the elliptical cross section, can be assessed by taking a top-view electron micrograph. The measurement of the long axis of the elliptical rods 
inside the structure was more difficult. We tried to obtain a side-view of the rods using ion-beam milling but this posed problems due to the melting of the polymer when exposed to the ion beam. To circumvent this problem, single rods were written with different laser powers between two large pillars, as shown in Figure S1, Supporting Information. This procedure allowed us to take SEM-images of the rods created by using different laser powers in DLW, from the top and at an inclined angle of $45^{\circ}$. The short and long axis were measured several times at different locations. The FWHM of the grey value profile of a line perpendicular to the rod was taken to determine the size. A mean value was obtained by measuring the size on different positions on the same image and we get a standard deviation of about $7 \%$, which is attributed to the uneven surface of the rods and the limited accuracy of the procedure. The results are summarized in Table S1, Supporting Information.

Absorption Spectrum of the IP-Dip Polymer: Figure S2, Supporting Information shows the absorption spectrum for a glass substrate coated with a $L=50 \mu \mathrm{m}$ and $L=100 \mu \mathrm{m}$ layer of developed IP-Dip photoresist. The effective thickness of the woodpile crystals was $L_{\text {eff }}=2.96 \mu \mathrm{m}$ at a filling fraction of $35 \%$ for a total thickness of $t=8.5 \mu \mathrm{m}$. The absorbtion spectra of thick layers of photoresist $(L \geqslant 50 \mu \mathrm{m})$ were analyzed, since for micrometer thick homogeneous samples the signal was very weak and masked by strong Fabry-Perot oscillations. From the $L=50 \mu \mathrm{m}$ and $L=100 \mu \mathrm{m}$ measurement, the average attenuation coefficient $\mu$ was extracted using $T=\exp (-\mu L)$ and the expected transmittance for $L=L_{\text {eff }}$ was calculated (dashed green curve in Figure S2, Supporting Information). It was found that overall absorptive losses were small in the wavelength-band between $\lambda=0.9$ and $2.6 \mu \mathrm{m}$. Absorptive losses attenuate the transmitted power by less than $1.3 \%$ for a IP-Dip sample of thickness $L_{\text {eff. }}$.

Assessment of the Polymer Filling Fraction: The overlap between alternating layers of rods was taken into account in order to accurately estimate numerically the filling fraction of each sample. To this end, the unit cell of the digital representation of the woodpile structure was discretized, and every volume element (or voxel) belonging to at least one rod was labelled. Then the ratio between the labeled and unlabeled voxels was calculated to get the filling fraction. Using this method, $\phi_{0}$ was calculated for the unit cell of the perfect woodpile, but also $\phi_{0, d}$ for the unit cell containing a single defect. The parameters of the rods were taken from the rod size measurements (see Table S1, Supporting Information). For a sample having a defect density $\rho$, the filling fraction of the sample was then $\phi=\left(1-\rho V_{0}\right) \phi_{0}+\rho V_{0} \phi_{0, d}$ where $V_{0}$ is the volume of the unit cell.

Band Structure Calculations: The woodpile has a FCC lattice, see Figure S3, Supporting Information. Its band structure was calculated in the first Brilloin zone (see figure S5, Supporting Information for the geometry). Usually, the (pseudo)gap was observed in the $\Gamma-X_{z}$ direction. Because of the Cassegrain objective, transmission of light having an incident angle between $15^{\circ}$ and $30^{\circ}$ was measured. The measurements were therefore compared with band structure calculations for the same angles. To achieve this, calculations of the band structure were performed in the $\Gamma-\mathrm{P}_{\theta}(\varphi)$ directions as a function of the azimuthal angle $\varphi$ for a fixed polar angle of $\theta=\arcsin \left(\theta_{\text {mean }} / n_{\text {eff }}\right)$ with $\theta_{\text {mean }}=\left(15^{\circ}+30^{\circ}\right) / 2$ due to the Cassegrain objective and $n_{\text {eff }}(\phi)$ calculated with the Maxwell-Garnett mixing formula ${ }^{[38]}$ (See Figure S5, Supporting Information and its legend for the geometry).

Figure S4, Supporting Information shows the band structure of the perfect crystal (elliptical rods, $a=0.42 \mu \mathrm{m}, b=0.15 \mu \mathrm{m}, d=1.2 \mu \mathrm{m}$ ) for different $\Gamma-\mathrm{P}_{\theta}(\phi)$ directions. $\theta$ is constant and $\phi$ is equally distributed between 0 and $90^{\circ}$ (for symmetry reasons, there was no need to calculate for all angles between 0 and $360^{\circ}$ because the structure is invariant by a rotation of $90^{\circ}$ around the $\hat{z}$ axis). The bandgap corresponds to the intersection of the bandgaps calculated for $\phi \in\left[0,90^{\circ}\right]$.

Beer-Lambert's Law: Beer-Lambert's law, Equation (1), was used to calculate the light scattering mean free path for a fixed height of structures. One could argue that a more precise way to determine the light scattering mean free path would be to measure $T$ at different heights and fit $\ell_{\mathrm{s}}$ to $\ln T=-L / \ell_{\mathrm{s}}$. However, doing so would require a vast number of samples, and thus we decided to measure different heights of structures for one defect rate only. Moreover, L-dependent measurements are not necessarily more accurate. Measurements at small $L$ are less precise due to a lack of scattering. Results for sample thicknesses $L>10 \mu \mathrm{m}$ can be affected by additional defect scattering due to strain-induced deformations, commonly observed in DLW. For a comparison, we first determine $\ell_{\mathrm{s}}$ for our standard fixed height (red to green lines of Figure S6, Supporting Information). Next, we obtain $\ell_{\mathrm{s}}$ from a linear fit to $T=-L / \ell_{\mathrm{s}}$ for each wavelength (inset of Figure S6, Supporting Information shows the data points and the fit at $\left.\lambda_{0}=1.95 \mu \mathrm{m}\right)$. We generally find good agreement between the fit to In $T=-L / \ell_{\mathrm{s}}$ and the $\ell_{\mathrm{s}}$ values calculated using just one height $L$.

We have verified that the spectral variation of the absorption coefficient can be safely neglected. We can write $T(L)+R(L)=\alpha^{\prime} \exp (-\bar{\mu} L) \exp \left(-L / \ell_{\mathrm{s}}\right)$ with $\alpha \equiv \exp (-\bar{\mu} L) \alpha^{\prime}$. We find that variation of $\bar{\mu}(\lambda)$ for $L_{\text {eff }}=2.96 \mu \mathrm{m}$ is very small and its influence on $T(\lambda)$ amounts to only a fraction of a percent and is thus negligible as shown in Figure S7, Supporting Information.

Rayleigh-Gans-Debye Scattering of a Hollow Cylinder: The form factor of a finite cylinder of radius $r$ and length $d$ (see Figure S8, Supporting Information for the geometry), illuminated by a beam making an angle $\zeta$ with its axis oriented along the $\hat{z}$ axis, as found in Bohren and Huffman, ${ }^{[46]}$ is

$f_{c}=\frac{1}{\pi r^{2} d} \int_{-d / 2}^{d / 2} e^{-i k A z} d z \int_{0}^{r} \rho d \rho \int_{0}^{2 \pi} e^{-i k \rho(B \cos \psi+C \sin \psi)} d \psi$

The integration is done in cylindrical coordinates $(\rho, \psi, z)$, for which the $\hat{z}$ axis is oriented along the direction of the cylinder. Note that in this calculation, to take advantage of the radial symmetry, the cylinders are oriented along the $\hat{z}$ axis, whereas in the rest of the paper they are perpendicular to the $\hat{z}$ axis. $k=2 \pi n_{\text {eff }} / \lambda_{0}$ (where $n_{\text {eff }}$ is the effective refractive index of the medium, and $\lambda_{0}$ the wavelength in vacuum of the incoming beam) is the wave number. Finally,

$A=\cos \zeta+\sin \theta \cos \varphi$

$B=\sin \theta \sin \varphi$

$C=\cos \theta-\sin \zeta$

$M=\sqrt{B^{2}+C^{2}}$

where $\theta \in[0, \pi]$ and $\varphi \in[0,2 \pi]$ are the spherical coordinates angles for scattered wave.

To calculate the form factor of a hollow cylinder, one just has to perform the second integration between $r_{1}$ and $r_{2}$ (the inner and outer radii of the cylinder) instead of 0 and $r$. Performing all the integration,

$f_{\text {hc }}(\theta, \varphi ; \zeta)=\frac{2}{d\left(r_{2}^{2}-r_{1}^{2}\right)} \frac{r_{2} J_{1}\left(k r_{2} M\right)-r_{1} J_{1}\left(k r_{1} M\right)}{k M} \frac{2 \sin (k A d / 2)}{k A}$

is obtained, where $J_{1}$ is the Bessel function of the first kind of order 1.

The RGD scattering cross section for a hollow cylinder making an angle of $\zeta$ with respect to the incoming beam is then defined through the integral over all solid angles $\Omega$

$C_{\mathrm{sca}, \mathrm{hc}}(\zeta)=\int_{4 \pi} \frac{k^{4}}{4 \pi^{2}}(m-1)^{2} v^{2}\left|f_{\mathrm{hc}}(\theta, \varphi ; \zeta)\right|^{2}\left[\cos ^{2} \theta \cos ^{2} \varphi+\sin ^{2} \varphi\right] d$

The integrations over $\theta$ and $\varphi$ were performed numerically.

In this paper, the incoming light made an angle $\zeta=\frac{\pi}{2}-\theta_{\text {eff }}$ with the axis of the cylinders, where $\theta_{\text {eff }}=\arcsin \left(\theta_{\text {mean }} / n_{\text {eff }}\right)$ and $n_{\text {eff }}$ should depend on the filling fraction. Because each curve and point plotted in the main text Figure 7 was deduced from measurements done varying the filling fraction, $n_{\text {eff }}$ was therefore kept a constant and is equaled to the value of the Maxwell-Garnett refractive index of the perfect structure. 


\section{Supporting Information}

Supporting Information is available from the Wiley Online Library or from the author.

\section{Acknowledgements}

The authors thank Luis Froufe-Pérez for fruitful discussions. The authors acknowledge financial support by the Swiss National Science Foundation under grants No. 169074 and 188494 . This work benefited from support from the Swiss National Science Foundation through the National Center of Competence in Research Bio-Inspired Materials, No. 182881.

\section{Conflict of Interest}

The authors declare no conflict of interest.

\section{Keywords}

light scattering, optical defects, photonic crystals

Received: October 2, 2020 Revised: December 3, 2020 Published online:

[1] J. Joannopoulos, S. Johnson, J. Winn, R. Meade, Photonic Crystals: Molding the Flow of Light, 2nd ed., Princeton University Press, Princeton, NJ 2008.

[2] R. J. Spry, D. J. Kosan, Appl. Spectrosc. 1986, 40, 782.

[3] J. F. Galisteo-López, E. Palacios-Lidón, E. Castillo-Martínez, C. López, Phys. Rev. B 2003, 68, 115109.

[4] C. Marichy, N. Muller, L. S. Froufe-Pérez, F. Scheffold, Sci. Rep. 2016, 6, 21818

[5] K. Ho, C. Chan, C. Soukoulis, R. Biswas, M. Sigalas, Solid State Commun. 1994, 89, 413.

[6] P. Russell, Science 2003, 299, 358

[7] M. Hochberg, T. Baehr-Jones, Nat. Photonics 2010, 4, 492.

[8] E. C. Nelson, N. L. Dias, K. P. Bassett, S. N. Dunham, V. Verma, M. Miyake, P. Wiltzius, J. A. Rogers, J. J. Coleman, X. Li, P. V. Braun, Nat. Mater. 2011, 10, 676.

[9] J. E. Wijnhoven, W. L. Vos, Science 1998, 281, 802.

[10] P. V. Braun, P. Wiltzius, Nature 1999, 402, 603.

[11] M. Campbell, D. N. Sharp, M. T. Harrison, R. G. Denning, A. J. Turberfield, Nature 2000, 404, 53.

[12] M. Deubel, G. von Freymann, M. Wegener, S. Pereira, K. Busch, C. M. Soukoulis, Nat. Mater. 2004, 3, 444.

[13] C. López, Adv. Mater. 2003, 15, 1679.

[14] S.-H. Nam, J. Park, S. Jeon, Adv. Funct. Mater. 2019, 29, 1904971.

[15] Y. A. Vlasov, V. N. Astratov, A. V. Baryshev, A. A. Kaplyanskii, O. Z. Karimov, M. F. Limonov, Phys. Rev. E 2000, 61, 5784.

[16] A. F. Koenderink, W. L. Vos, Phys. Rev. Lett. 2003, 91, 213902.
[17] J. Huang, N. Eradat, M. E. Raikh, Z. V. Vardeny, A. A. Zakhidov, R. H. Baughman, Phys. Rev. Lett. 2001, 86, 4815.

[18] A. Ledermann, L. Cademartiri, M. Hermatschweiler, C. Toninelli, G. A. Ozin, D. S. Wiersma, M. Wegener, G. von Freymann, Nat. Mater. 2006, 5, 942

[19] C. M. Soukoulis, M. Wegener, Nat. Photonics 2011, 5, 523.

[20] S. E. Skipetrov, Nature 2004, 432, 285.

[21] M. Florescu, S. Torquato, P. J. Steinhardt, Appl. Phys. Lett. 2010, 97, 201103.

[22] L. S. Froufe-Pérez, M. Engel, J. J. Sáenz, F. Scheffold, Proc. Natl. Acad. Sci. USA 2017, 114, 9570.

[23] S. N. Fernandes, Y. Geng, S. Vignolini, B. J. Glover, A. C. Trindade, J. P. Canejo, P. L. Almeida, P. Brogueira, M. H. Godinho, Macromol. Chem. Phys. 2013, 214, 25.

[24] F. Pratesi, M. Burresi, F. Riboli, K. Vynck, D. S. Wiersma, Opt. Express 2013, 21, A460.

[25] G. J. Aubry, L. S. Froufe-Pérez, U. Kuhl, O. Legrand, F. Scheffold F. Mortessagne, Phys. Rev. Lett. 2020, 125, 127402.

[26] S. John, Phys. Rev. Lett. 1987, 58, 2486.

[27] K. Ishizaki, M. Koumura, K. Suzuki, K. Gondaira, S. Noda, Nat. Photonics 2013, 7, 133.

[28] E. Yablonovitch, Phys. Rev. Lett. 1987, 58, 2059.

[29] A. F. Koenderink, A. Lagendijk, W. L. Vos, Phys. Rev. B 2005, 72, 153102.

[30] V. Astratov, A. Adawi, S. Fricker, M. Skolnick, D. Whittaker, P. Pusey, Phys. Rev. B 2002, 66, 165215.

[31] P. D. García, R. Sapienza, C. Toninelli, C. López, D. S. Wiersma, Phys. Rev. A 2011, 84, 023813.

[32] H.-B. Sun, S. Matsuo, H. Misawa, Appl. Phys. Lett. 1999, 74, 786.

[33] I. Staude, M. Thiel, S. Essig, C. Wolff, K. Busch, G. von Freymann, M. Wegener, Opt. Lett. 2010, 35, 1094

[34] I. Staude, G. von Freymann, S. Essig, K. Busch, M. Wegener, Opt. Lett. 2011, 36, 67.

[35] P. Mueller, M. Thiel, M. Wegener, Opt. Lett. 2014, 39, 6847.

[36] S. Dottermusch, D. Busko, M. Langenhorst, U. W. Paetzold, B. S. Richards, Opt. Lett. 2019, 44, 29.

[37] S. G. Johnson, J. D. Joannopoulos, Opt. Express 2001, 8, 173.

[38] J. C. M. Garnett, Philos. Trans. R. Soc. London A: Math., Phys. 1904, 203, 385.

[39] P. D. García, R. Sapienza, L. S. Froufe-Pérez, C. López, Phys. Rev. B 2009, 79, 241109.

[40] F. García-Santamaría, J. F. Galisteo-López, P. V. Braun, C. López, Phys. Rev. B 2005, 71, 195112.

[41] D. J. Pine, D. A. Weitz, J. X. Zhu, E. Herbolzheimer, J. Phys. France $1990,51,2101$

[42] N. W. Ashcroft, N. D. Mermin, Solid State Physics, Harcourt College Publishers, New York 1976.

[43] S. Fraden, G. Maret, Phys. Rev. Lett. 1990, 65, 512.

[44] R. Rezvani Naraghi, S. Sukhov, J. J. Sáenz, A. Dogariu, Phys. Rev. Lett. 2015, 115, 203903.

[45] G. J. Aubry, L. Schertel, M. Chen, H. Weyer, C. M. Aegerter, S. Polarz, H. Cölfen, G. Maret, Phys. Rev. A 2017, 96, 043871.

[46] C. F. Bohren, D. R. Huffman, Absorption and Scattering of Light by Small Particles, Wiley, New York 1998.

[47] T. Sperling, L. Schertel, M. Ackermann, G. J. Aubry, C. Aegerter G. Maret, New J. Phys. 2016, 18, 013039.

[48] N. Muller, J. Haberko, C. Marichy, F. Scheffold, Optica 2017, 4, 361. 